\title{
Long-Term Monitoring and Identification of Bridge Structural Parameters
}

\author{
Serdar Soyoz \\ MMI Engineering, Oakland, CA, USA \\ (Formerly Department of Civil and Environmental Engineering, University of California, Irvine, CA, USA) \\ $\&$ \\ Maria Q. Feng* \\ Department of Civil and Environmental Engineering, University of California, Irvine, CA, USA
}

\begin{abstract}
Vibration of a new concrete bridge was monitored and change in the bridge structural stiffness was identified accordingly over a 5-year period. This threespan 111-m long bridge is instrumented with 13 acceleration sensors at both the superstructure and the columns. The sensor data are transmitted to a server computer wirelessly. Modal parameters of the bridge, that is, the frequencies and the modal shapes were identified by processing 1,707 vibration data sets collected under traffic excitations, based on which the bridge structural parameters, stiffness and mass, and the soil spring values were identified by employing the neural network technique. The identified superstructure stiffness at the beginning of the monitoring was $97 \%$ of the stiffness value based on the design drawings. In the identified modal frequencies, a variation from $-10 \%$ to $+10 \%$ was observed over the monitoring period. In the identified stiffness values of the bridge superstructure, a variation from $-3 \%$ to $+3 \%$ was observed over the monitoring period. Based on the statistical analysis of the collected data for each year, $5 \%$ decrease in the first modal frequency and $2 \%$ decrease in the superstructure stiffness were observed over the 5-year monitoring period. Probability density functions were obtained for stiffness values each year. Stiffness threshold
\end{abstract}

\footnotetext{
*To whom correspondence should be addressed. E-mail: mfeng@
} uci.edu. values for the collapse of the bridge under the operational loading can be determined. Then the number of years can be assessed for which the area under the proposed probability density functions is greater than the threshold value. So the information obtained in this study is valuable for studying aging and long-term performance assessment of similar bridges.

\section{INTRODUCTION}

The sophisticated highway system in the United States is supported by tens of thousands of bridges and viaducts. Lack of information about the health condition of these bridges can cause safety hazards to the traveling public, halt mobility of the transportation network, and disrupt the emergency response. The current practice relies on visual inspection for damage detection, which is time consuming, insufficient, subjective, and requires the physical presence of the crew on the structure that is potentially hazardous after major natural or man-made events such as earthquakes and accidents.

The frequency of visual inspection and the qualification of the inspectors were regulated by the National Bridge Inspection Standards (1996). The Federal Highway Administration (FHWA) Recoding and Coding 
Guide (FHWA, 1995) also provides guidance in terms of the condition ratings and the documentation in current practice. Even with these provisions, a recent investigation initiated by FHWA to examine the reliability of the visual inspections reveals significant variability in the structural condition assessments by the inspectors (Phares et al., 2004). Moreover, visual inspection cannot quantitatively evaluate the strength and/or the deformation capacity reserve of a bridge. The LongTerm Bridge Performance Program was recently initiated by FHWA, exploring sensor-based continuous monitoring of bridges under the traffic conditions as well as during the extreme events such as earthquakes (http://www.tfhrc.gov/structur/ltbp.htm).

Sensor-based structural health monitoring can revolutionize the way of inspecting structures in a rapid, remote, automated, and objective fashion. By installing appropriate sensors at the critical locations on a bridge structure, transmitting the sensor data through a communications network, and analyzing the data through a software platform, structural damage and deterioration of the bridge can be automatically, remotely, and rapidly assessed, without sending inspection crews to the site.

System identification methods for structures based on vibration measurement can be found extensively in the literature (e.g., Beck and Jennings, 1980; Safak, 1989; Safak, 1991; Ghanem and Shinozuka, 1995; Shinozuka and Ghanem, 1995; Doebling et al., 1996). These methods can be grouped into two categories depending on whether the identification is carried out in the frequency or in the time domain. The frequency domain methods basically identify the changes in the modal frequencies, damping ratios, or modal shapes based on the vibration measurement. Elemental stiffness values can then be further identified by minimizing the errors between the modal parameters identified from the vibration measurement and finite element (FE) analysis. The neural network technique (e.g., Levin and Lieven, 1992; Feng and Bahng, 1999; Masri et al., 2000; Yun et al., 2001; Huang and Loh, 2001; Feng et al., 2003; Lee et al., 2005) can be used to obtain the structural elemental stiffness values based on the identified modal parameters of a structure including the modal frequencies and the modal shapes. Radial basis function neural network applications in infrastructure engineering have been reported (e.g., Adeli and Karim, 2000; Karim and Adeli, 2002; Karim and Adeli, 2003; Adeli and Karim, 2005). Damage detection and control algorithms for structures using dynamic fuzzy wavelet neural network approaches have also been developed (Jiang and Adeli, 2007; Jiang and Adeli, 2008a, 2008b). The main advantage of the neural network technique is its realtime identification capability. The mapping between the modal parameters (input) and the stiffness values (output) is pre-trained and pre-tested and embedded into the network. This enables automatic processing of a vast amount of data on a regular basis to establish the stiffness database. Long-term stiffness database can reveal the trend in the structural aging process.

Deterioration of bridge decks has been studied by a number of researchers (e.g., Enright and Frangapol, 1998; Stewart and Rosowsky, 1998; Akgul and Frangapol, 2004). So far aging of the bridges has been determined based on the corrosion models and the Monte Carlo simulations without the monitoring data at bridges. This approach takes only the corrosion phenomenon into account but not the overall deterioration due to cracking or excessive vibration. Therefore information on the aging of a bridge structure based on longterm vibration monitoring of the bridge would fill an important gap in the current structural health monitoring research. Changes in the modal frequency and structural parameters based on a limited number of vibration measurements in a 2-year period for two bridges were reported (e.g., Choi et al., 2004; Guan et al., 2006). Still limited literature exists on a longer-term and continuous monitoring of bridge structures.

In this study, a database for both the modal parameters and stiffness values of a new concrete highway bridge has been established based on 1,707 sets of traffic-excited vibration data over a 5-year period. The stiffness values were identified using the neural network-based system identification technique. Identified stiffness values can be further used for the condition assessment of the bridge superstructure.

\section{BRIDGE AND MONITORING SYSTEM}

Figure 1 shows the new bridge monitored in this study, Jamboree Road Over-crossing (JRO). It is a three-span continuous cast-in-place pre-stressed

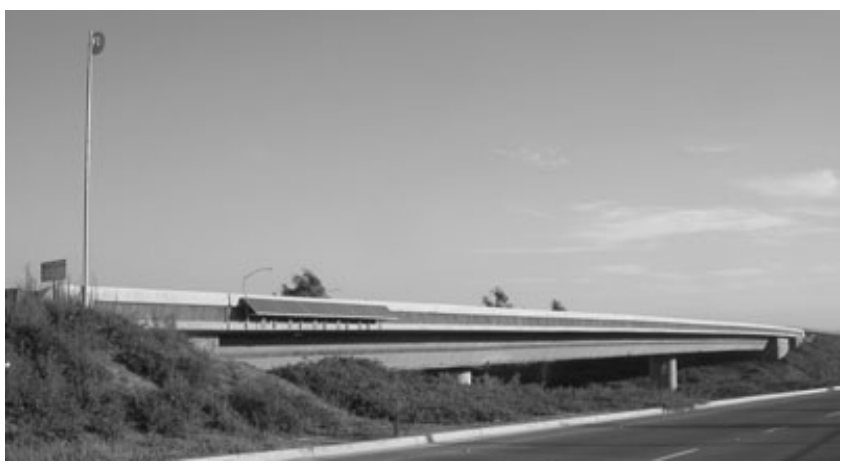

Fig. 1. Jamboree Road Over-crossing. 
post-tensioned box-girder bridge located in Irvine, California. The total length of the bridge is $111.9 \mathrm{~m}$ with each span length of $35.5,46.1$, and $30.3 \mathrm{~m}$. The bridge is supported on two monolithic single columns and sliding bearings on both abutments. The sliding bearings allow creep, shrinkage, and thermal expansion or contraction. The bridge was opened to traffic in 1998 and the monitoring of the bridge started in 2002.

\subsection{Sensor layout}

In total, 13 servo-type accelerometers were installed on the bridge by the second author and her research team 4 years after the bridge was opened to traffic. Figure 2 shows the locations of the accelerometers. The accelerometers on the superstructure were placed along the center line of the bottom of the girder. The accelerometers were also installed on the top and bottom of column 3. In addition, a displacement sensor was installed at abutment 4 to measure the movement of the superstructure with respect to the abutment due to shortening, creep, shrinkage, as well as seismic excitations. The sensors are connected to a data logger located at the bridge site. Solar panels were also installed to provide backup power supply to the sensor system.

\subsection{Wireless remote data acquisition system}

A wireless data acquisition system using point-to-point antennas was developed for remotely controlling the sensor system and acquiring sensor data at a research center located at the University of California, Irvine (UCI), and 6 miles away from the bridge site. Soft- ware platform was developed and installed on a computer at the UCI campus, which functions as a server to receive streaming data from the data logger on the remote bridge site, and to save and buffer it for Internet publication. The software platform has an algorithm to accommodate data transmission errors during wireless communication. Besides this server software, a Java applet was further developed in this project for displaying real-time data on Internet. Figure 3 displays the waveforms of the data in the buffer of the server. It is available at http://mfeng.calit2.uci.edu/ (Special approval from the bridge owner is needed for downloading the data). This pair of server/client software also provides a way to check the working status of the JRO monitoring system.

\section{IDENTIFICATION OF MODAL PARAMETERS}

Over a 5-year period in this bridge's life (2002-2006), in total 1,707 traffic-induced vibration data sets have been collected. The monitoring system is automatically triggered when the vibration level exceeds a threshold and 1 minute long data is recorded. This section first presents identification of modal parameters based on the collected vibration data and then a statistical analysis of the data. Because traffic load induces mostly vertical vibration of the superstructure (as shown in Figure 4), only the data from the accelerometers in the vertical direction on the superstructure were used in the analysis. Consistent data quality over a 5-year monitoring period has been observed. Sensors 15 and 16 were not used due to their malfunctioning.

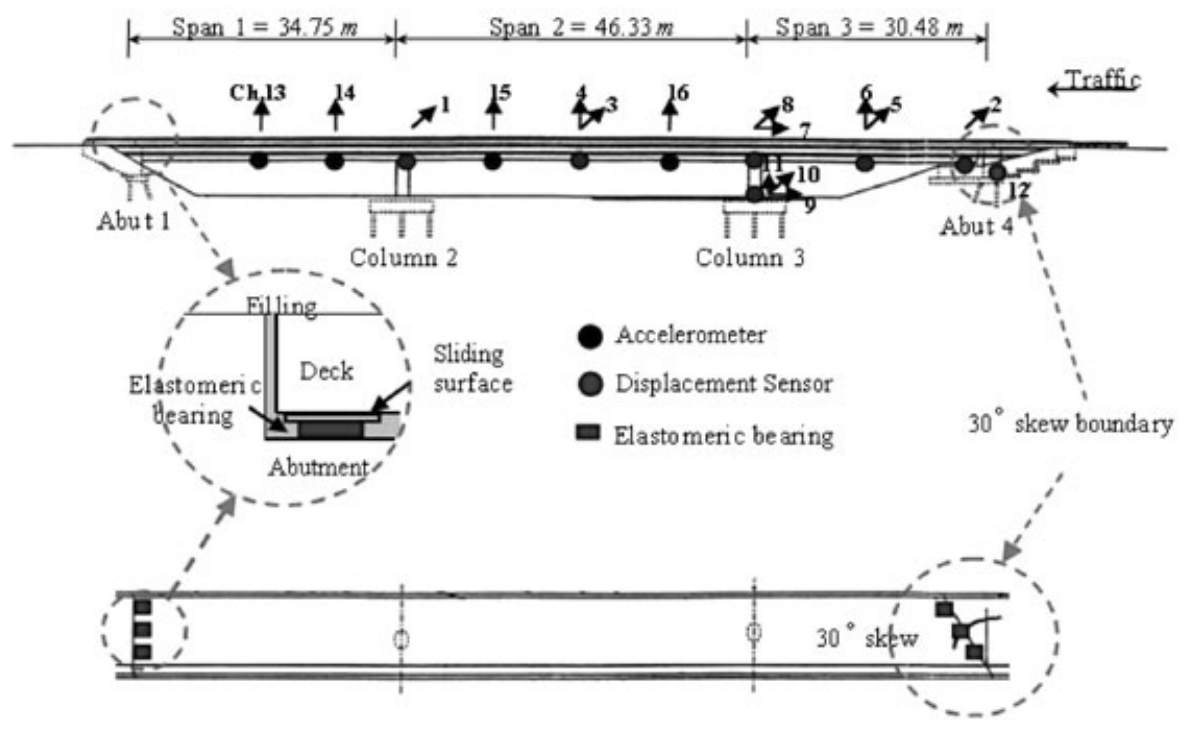

Fig. 2. Sensor layout on JRO. 


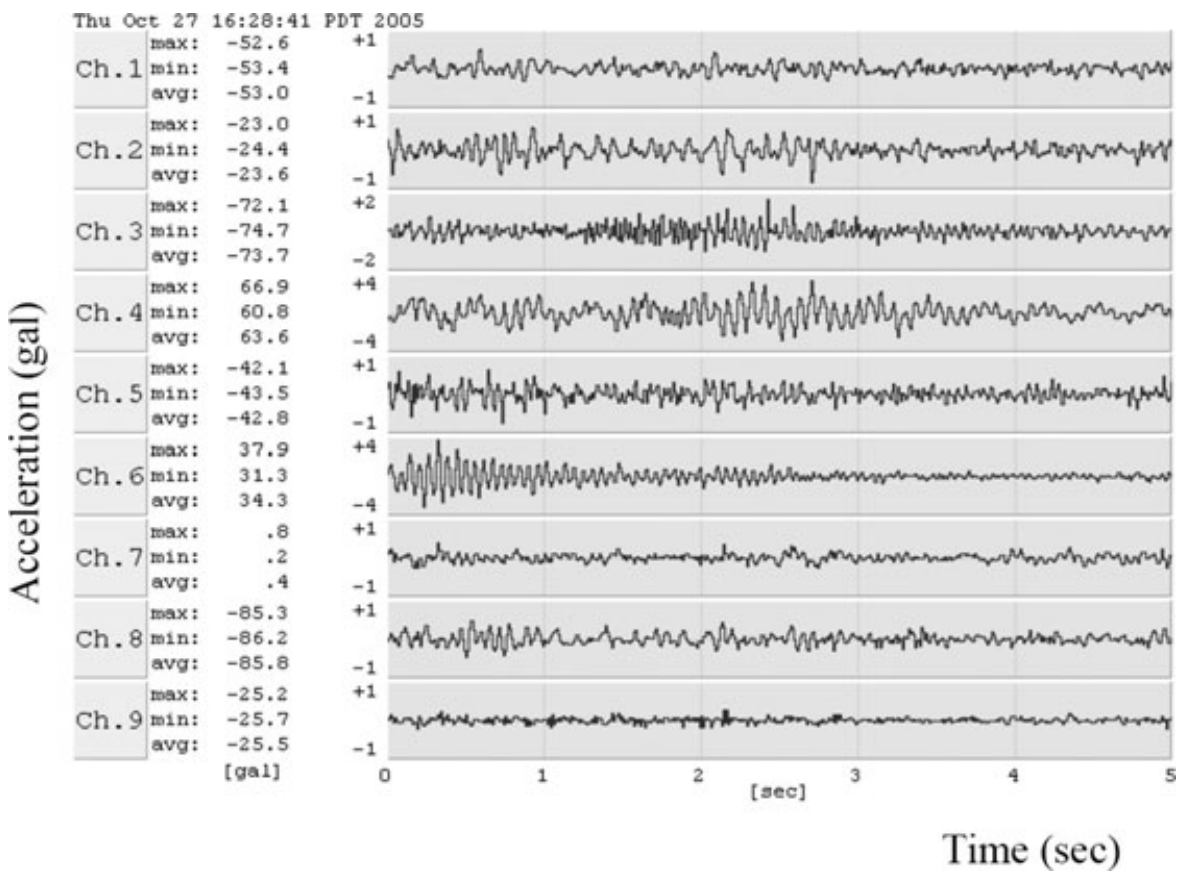

Fig. 3. Real-time sharing of monitoring data on Internet.
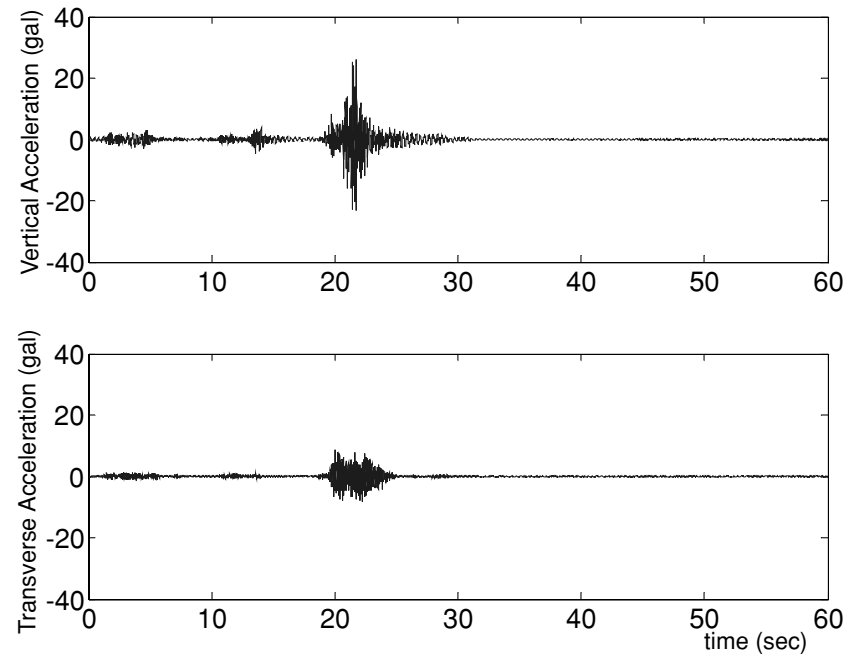

Fig. 4. Traffic-induced vibrations at JRO.

\subsection{Method for modal parameter identification}

Figure 4 shows typical acceleration time histories measured in the middle of span 2 in the vertical and transverse directions. It was confirmed that the $99 \%$ of the 1,707 response measurements, vertical vibration level in the middle of the deck, was lower than 50 gal. Each data set was 1 minute long and sampled at $100 \mathrm{~Hz}$. These 1 minute data were divided into segments and processed with $0.025 \mathrm{~Hz}$ frequency resolution. Hanning window was employed for each segment. Consecutive segments were overlapped $50 \%$ in frequency domain to smooth the results further. The vibration amplitudes in the transverse direction were observed to be less than half of that in the vertical direction, indicating traffic loads mainly induce vibrations in the vertical direction for a straight bridge. The vibration amplitudes in the transverse direction were observed to be $70 \%$ of those in the vertical direction for a curved bridge (Feng et al., 2006).

For traffic-induced vibration, the input loads are difficult, if not impossible, to measure, and thus the dynamic characteristics are estimated based on the responses only. The frequency domain decomposition (FDD) method (e.g., Brinker et al., 2001; Otte et al., 1990) was used to extract modal parameters from the vibration measurements without requiring information about the input loads. The FDD method is also capable of identifying closely coupled modes, thus obtaining better estimates (Otte et al., 1990). In this method, taking the singular value decomposition, the spectral density matrix $S_{Y Y}(w)$ of the response vector $\boldsymbol{Y}(\boldsymbol{t})$ is decomposed into

$$
S_{Y Y}(w)=U(w) \cdot \Sigma(w) \cdot U^{H}(w)
$$

where $\Sigma(w)=$ diagonal matrix of the singular values; $U(w)=$ unitary matrix of the singular vectors; and the superscript $H$ denotes the complex conjugate and transpose. It has been shown by (Otte et al., 1990) that, when 

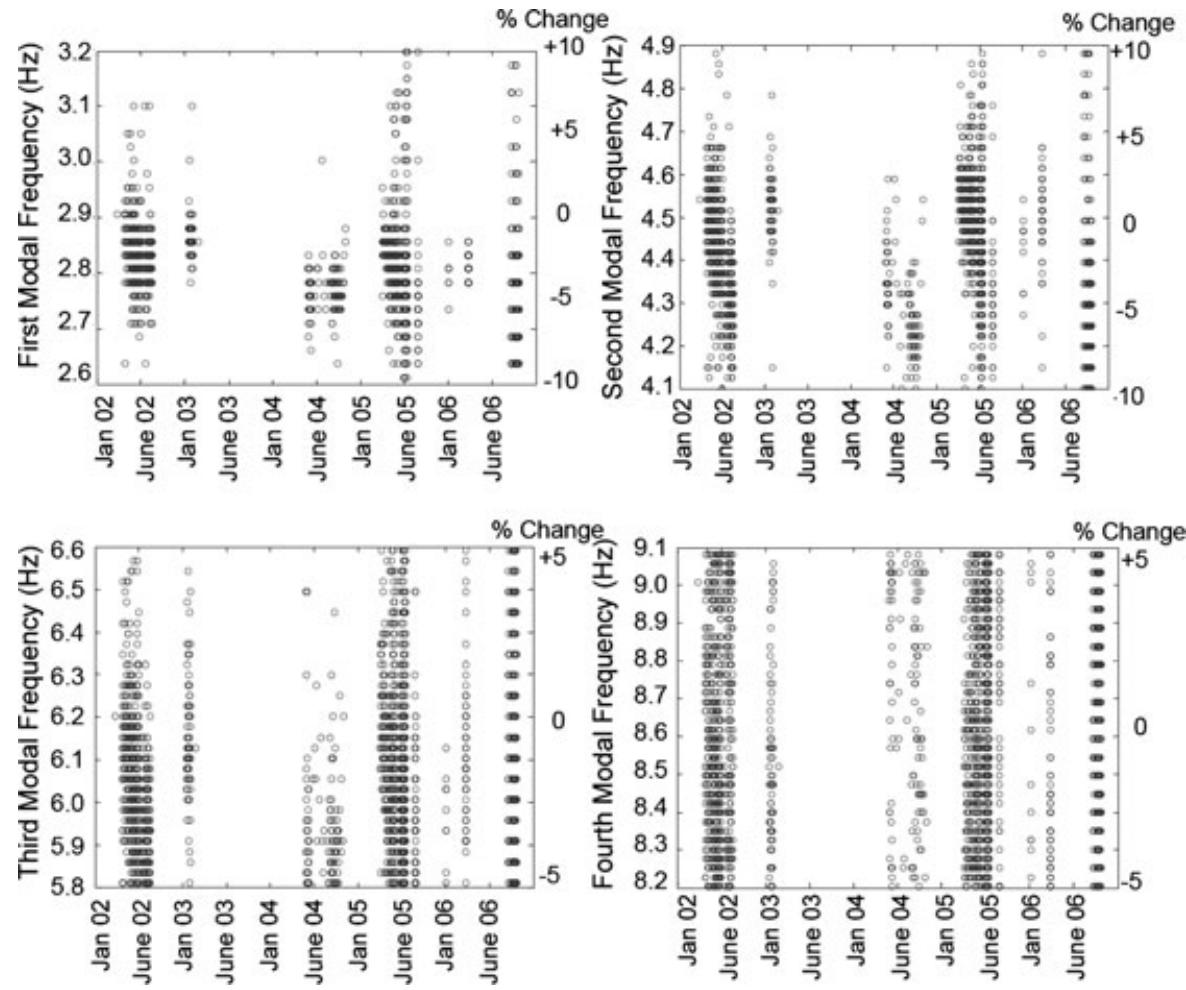

Fig. 5. Modal identification results.

the structure is loaded with the broadband excitation, near the modal frequencies, $\Sigma(w)$ contains a set of functions that are approximations of the auto-spectral density functions of the modes' equivalent single degreeof-freedom (SDOF) systems in the normal coordinates, while the vectors in $U(w)$ are the modal shapes of the corresponding modes.

From the traffic-induced ambient vibration data sets, the modal frequencies and the modal shapes were extracted using the FDD method. Figure 5 shows the variations of the first four modal frequencies over the 5-year monitoring period. As seen in the figure, the variation in the identified modal frequencies is in the order of $+/-10 \%$ of that obtained in the very beginning of the monitoring.

\subsection{Statistical characteristics of the modal frequency}

High variation in the identified modal frequency was observed. This is considered due to two major reasons. The first reason is the influence of moving vehicles. The number of vehicles passing the bridge is different when the response is measured, that simply changes the total mass of the bridge structure. To investigate the influence of the vehicle mass, a parametric study was performed. Existence of 10 standard passenger vehicles changes total mass of the bridge in the order of $10 \%$ and the modal frequency of the bridge in the order of $5 \%$. Dynamic interaction between the vehicles and the bridge further increases the variation of the identified modal frequency (e.g., Green and Cebon, 1997; Yang and Yau, 1997; Pan and Li, 2002; Calcado et al., 2005). To avoid the influence of moving vehicles, ideally the modal identification should be performed by the nontransient part of the measured response data of the bridge. In other words, after the vehicle passes the bridge free vibration part in the signal should be used for the identification purposes. However, this is very difficult, if not impossible, under the operational loading due to the fact that almost always another vehicle enters to the bridge before the previous one leaves. Therefore the free vibration part of the data is not long enough for sufficient frequency resolution. In this study, the measured response of the bridge is analyzed in a probabilistic manner (to be discussed) to alleviate the influence of moving vehicles.

The second reason for the variation in the identified modal frequencies is known as the environmental effects such as the change in temperature and moisture. Variation in the identified modal parameters due to the temperature change was reported (e.g., Sohn et al., 1999; Peeters and DeRoeck, 2001; Feltrin, 2002). In this research temperature sensor was installed recently and no sufficient temperature data have been 
Table 1

Number of data points in each period

\begin{tabular}{lccccc}
\hline & 2002 & 2003 & 2004 & 2005 & 2006 \\
\hline Winter & 250 & 70 & 37 & 377 & 80 \\
Summer & 364 & - & 80 & 419 & 30 \\
\hline
\end{tabular}

recorded yet. Therefore, data collected in each year are grouped into two seasons: summer or winter. The average temperature for the City of Irvine is almost constant between November-April (referred to as winter) and May-October (referred to as summer). Table 1 shows the number of data points in each period.

In Table 1, it is clear that the number of data points is not uniformly distributed. Therefore a simple best fit to all of the identified modal values is not reliable to obtain a trend over time. To interpret the data in a more quantitative manner, probability distributions were fitted to the modal identification results for each season. Figure 6 shows the Beta fit to the histogram of the summer 2002 data set as an example. The reasons to choose Beta fit are explained in the following section.

Figure 7 shows the modes of each distribution for the summer and winter period over a 5-year period. Average of the best linear fits to summer and winter periods is given in Equation (2). Clear trend is observed indicating a decrease in the first modal frequency each year.

First Modal Frequency $=2.85-0.03 *$ years

Based on Equation (2), the bridge's first modal frequency decreases $0.03 \mathrm{~Hz}$ per year, which constitutes $5 \%$ reduction over a 5 -year period. Figure 7 also shows

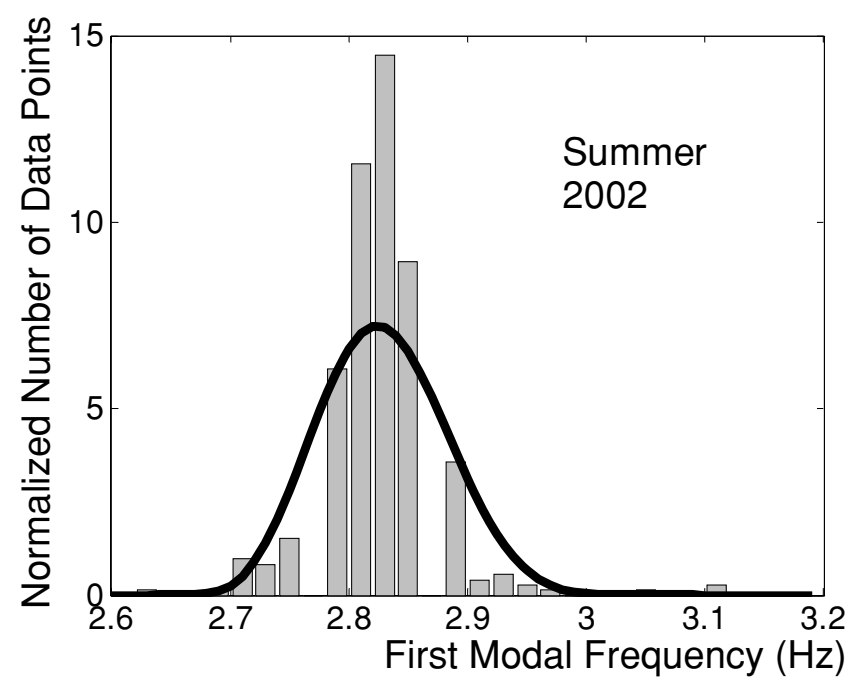

Fig. 6. Probability distribution of first modal frequency.
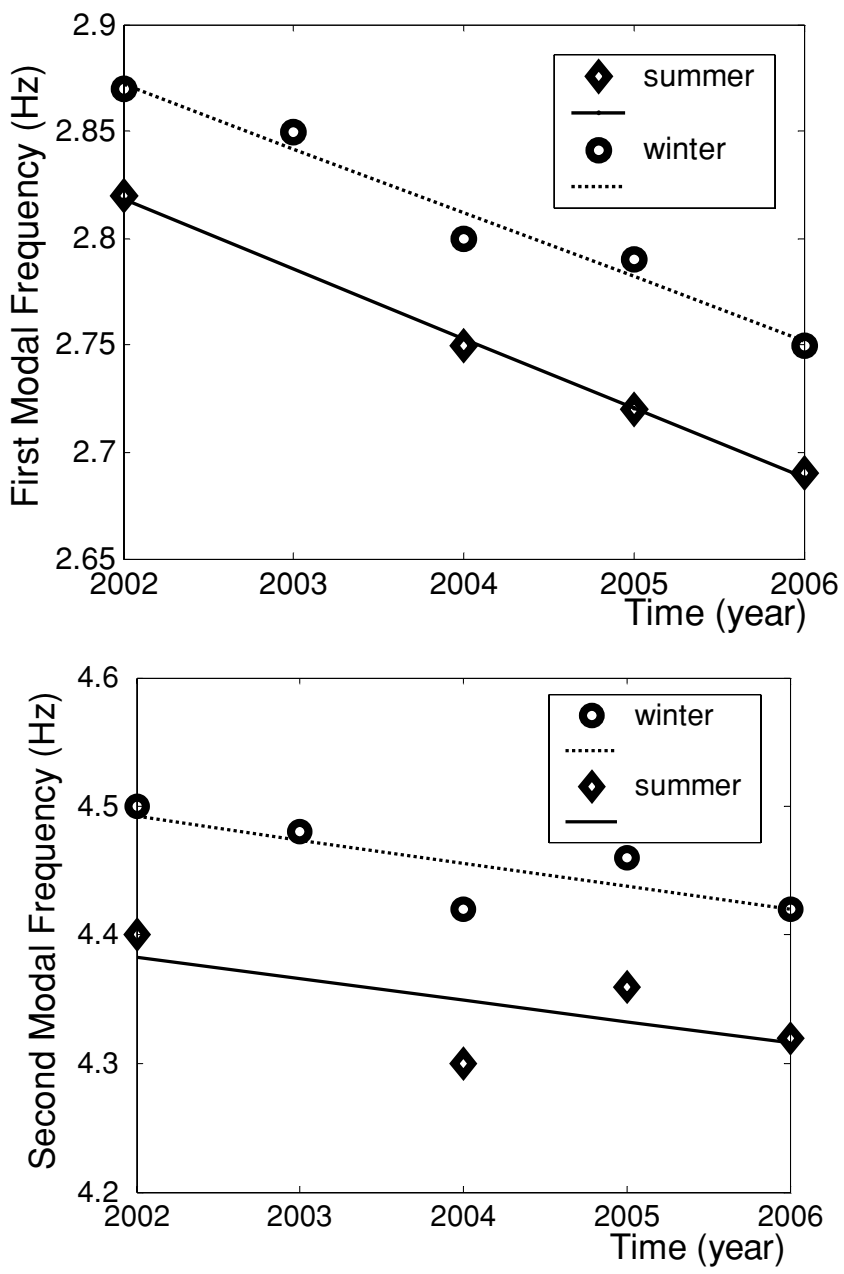

Fig. 7. Variation of first and second modal frequencies.

the decrease in the second modal frequency. It was observed that the decrease in the second modal frequency was $2 \%$ over a 5 -year period.

Figure 8 shows the first modal shape identified based on the data collected in the very beginning of the period and that obtained from FE model. To investigate the change in the modal shape, similarity index between

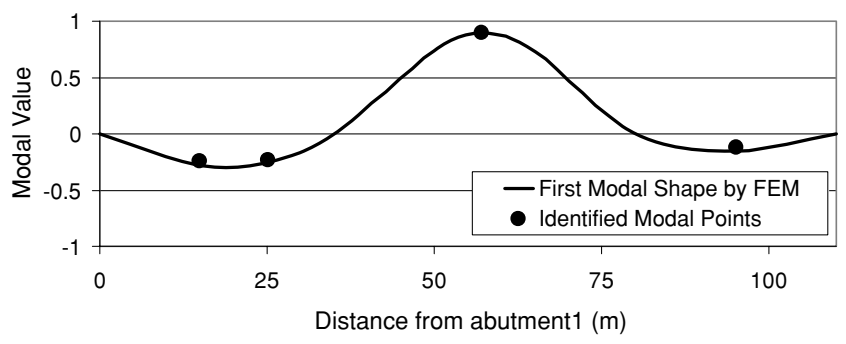

Fig. 8. First modal shape. 


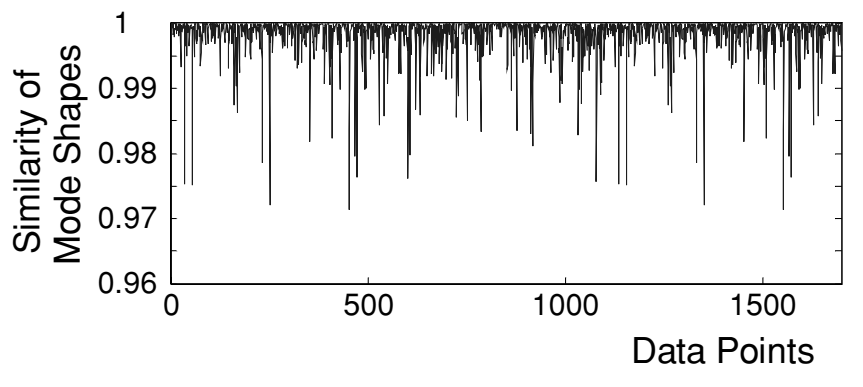

Fig. 9. Similarity of modal shapes.

the modal shapes identified in the very beginning of the monitoring and at any time is defined as follows:

$$
\operatorname{Similarity}\left(\Phi_{i}, \Phi_{j}\right)=\frac{\left(\Phi_{i}^{T} \Phi_{j}\right)^{2}}{\left(\Phi_{i}^{T} \Phi_{i}\right)\left(\Phi_{j}^{T} \Phi_{j}\right)}
$$

where $\Phi_{i}$ is the modal shape identified in the very beginning of the monitoring and $\Phi_{j}$ is the modal shape identified at any time afterwards.

Figure 9 plots the similarity index for the first modal shape over a 5-year period. It fluctuates between 0.98 and 1.00 without a clear trend. This implies that the decrease in the first modal frequency value is not due to damage at a specific location but due to the deterioration of the whole bridge structure over a 5-year period.

\section{IDENTIFICATION OF THE STRUCTURAL STIFFNESS}

Based on the identified modal parameters, the structural stiffness values were obtained using neural network-based identification. The natural frequencies and the structural parameters such as the stiffness and the mass of a multi-degree-of-freedom system cannot simply be correlated to each other as in a single-degreeof-freedom system. The effect of increasing mass value is not the same as the effect of reducing stiffness value in multi-degree-of-freedom systems. So the neural networks were applied for the structural parameter identification. This section first describes the identification method and the results, and then analyzes the identified stiffness values in a statistical way over a 5 -year monitoring period. Application of neural networks for identifying structural parameters of a TV tower and bridges under wind and earthquake excitations is also reported (Feng and Kim, 1998; Feng and Bahng, 1999; Feng et al., 2006).

\subsection{Finite element model}

A 3D preliminary FE model was developed for the bridge. The superstructure and columns were modeled
Table 2

Structural parameters for JRO

\begin{tabular}{lccccccc}
\hline & & \multicolumn{3}{c}{$\begin{array}{c}\text { Moment of } \\
\text { inertia }\left(m^{4}\right)\end{array}$} & & \multicolumn{2}{c}{$\begin{array}{c}\text { Spring } \\
\text { stiffness }\end{array}$} \\
\cline { 4 - 6 } \cline { 8 - 9 } Element & $\begin{array}{c}\text { Area } \\
\left(\mathrm{m}^{2}\right)\end{array}$ & $I_{X}$ & $I_{Y}$ & $I_{Z}$ & & $k_{d}$ & $k_{r}$ \\
\hline Deck & 5.94 & 7.63 & 3.01 & 5.94 & & N/A & N/A \\
Column & 3.53 & 2.51 & 0.72 & 1.51 & & N/A & N/A \\
Abutment & N/A & N/A & N/A & N/A & $2.0010^{8}$ & $7.5810^{8}$
\end{tabular}

$\mathrm{N} / \mathrm{A}=$ not available.

as 3D frame elements by using SAP2000. The crosssection area and the moment of inertia for each element were calculated from the design drawings and are listed in Table 2. The abutment was modeled as linear longitudinal and transverse rotational springs with the stiffness values determined based on the FHWA Recommendation (FHWA, 1996) listed in Table 2.

\subsection{Method for stiffness identification}

Radial basis function (RBF) networks were applied in this study to identify the structural parameters, namely, the structural stiffness, the abutment soil spring stiffness, and the bridge mass, based on the modal parameters extracted from vibration measurement. The RBF networks were chosen over the multi-layer back propagation networks due to the observations that the RBF networks were more stable. The relationship between input and output of a neural network can be nonlinear or linear, and its characteristics are determined by the weights assigned to the connections between the neurons in two adjacent layers. Changing these weights

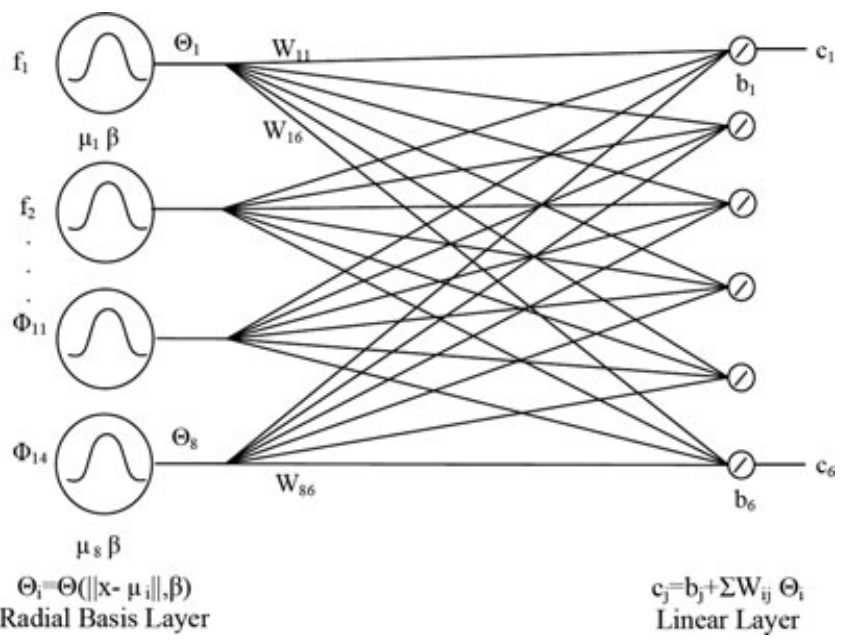

Fig. 10. Architecture of the neural network. 
will change the input-output relationship of the network.

Figure 10 shows the architecture of a RBF neural network. The overall response characteristics of a RBF network can be described by:

$$
c_{j}=b_{j}+\sum W_{i j} \Theta\left(\left\|x-\mu_{i}\right\|, \beta\right)
$$

where $x$ is the input vector, $\mu_{i}$ is the center of the $i$ th neuron, $\beta$ is the spread constant of the network, $\|\cdot\|$ is the Euclidean norm, $\Theta$ is the radial basis function of the network, typically a Gaussian function, $c_{i}$ is the output vector, $b_{j}$ is the bias of the $j$ th linear neuron, and $W_{i j}$ is the weight between the $i$ th $\mathrm{RBF}$ neuron and the $j$ th linear neuron.
The first four vertical modal frequencies and the first vertical modal shape represented at the four sensor locations 13-14-4-6 are the inputs to the neural network. The six correction coefficients for the six structural parameters, namely, the superstructure mass and stiffness, the column mass and stiffness, and the soil spring stiffness at abutments 1 and 4, are the outputs of the neural network. Training patterns were generated by extensive FE analysis with 10,000 sets of correction coefficients for the bridge. For training, the values of the structural stiffness, soil spring, and mass were varied from 0.2 to 1.2 times of those based on the design drawings. For each given set of these values, the corresponding frequencies were computed by FE analysis. Once the neural network was properly trained and tested, it was then used for identifying the correction coefficients of
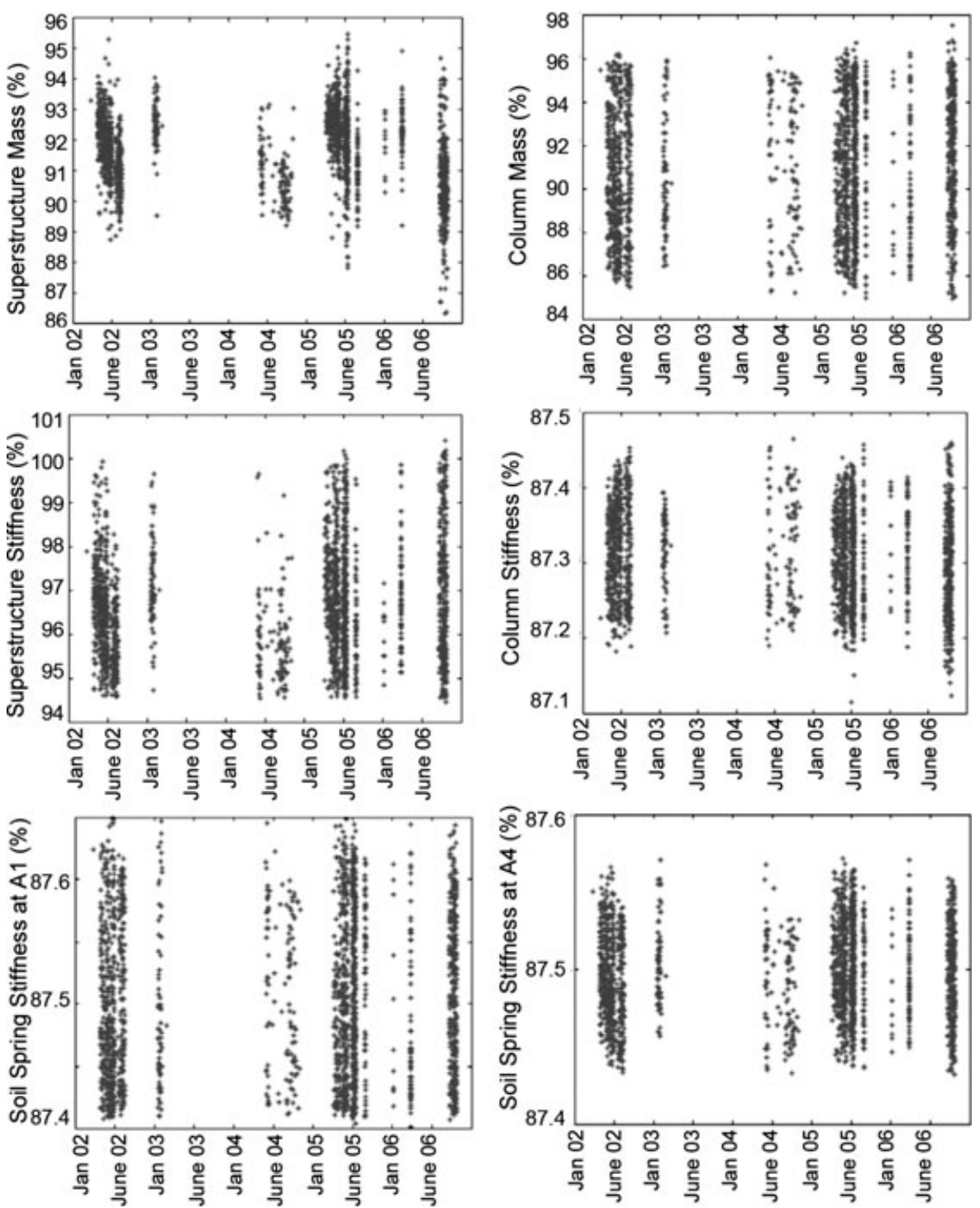

Fig. 11. Structural parameter identification results. 
the mass and stiffness based on the measured first four vertical modal frequencies and the first vertical modal shape.

The neural network was developed for the general purposes of identifying the change of structural properties (mass and stiffness) of both superstructure and columns of the bridge and the soil spring stiffness. For this reason, the six correction coefficients were chosen. Although this article focuses on the identification of the superstructure properties using only the traffic-induced vertical vibration, the same neural network can be used to identify the change of structural properties in the columns using horizontal vibration information such as earthquake responses. The identification using earthquake responses is beyond the scope of this study.

Figure 11 shows the correction coefficients of the bridge structural parameters and the soil spring stiffness identified based on the traffic-excited vibration data collected over a 5-year period. It is observed that the superstructure stiffness identified based on measurement is $97 \%$ of that obtained from the design drawings. In addition, there is a variation from $-3 \%$ to $+3 \%$ in the superstructure stiffness.

\subsection{Statistical characteristics of the stiffness}

Probability distributions were utilized to analyze the identified structural parameters in a more quantitative manner. Figure 12 shows the Normal probability plot; it is clear from the plot that Normal distribution does not fit to the data. To see the characteristic of the distribution, the histogram of the data was plotted. It was observed that the Beta distribution might be one of the candidates to represent the histograms. Figure 13 shows the Kolmogorov-Smirnov test for the Normal and the Beta distribution. Critical values at the $20 \%, 10 \%, 5 \%$, and $1 \%$ significance levels are $0.056,0.064,0.071$, and 0.085 , respectively. Therefore the Beta distribution is not rejected in any level whereas the Normal distribution is rejected in all the levels. Figure 14 shows the Beta fit to the histogram of the summer 2002 data set. The density function is given in Equation (5). $q$ and $r$ values for the distribution are 1.5 and 3.5, respectively, and standard deviation is 0.9 .

$$
\begin{aligned}
f_{X}(x) & =\frac{1}{B(q, r)} \frac{(x-a)^{q-1}(b-x)^{r-1}}{(b-a)^{q+r-1}} & & a \leq x \leq b \\
& =0 & & \text { elsewhere }
\end{aligned}
$$

in which $q$ and $r$ are parameters of the distribution, and $B(q, r)$ is the beta function:

$$
B(q, r)=\int_{0}^{1} x^{q-1}(1-x)^{r-1} d x
$$

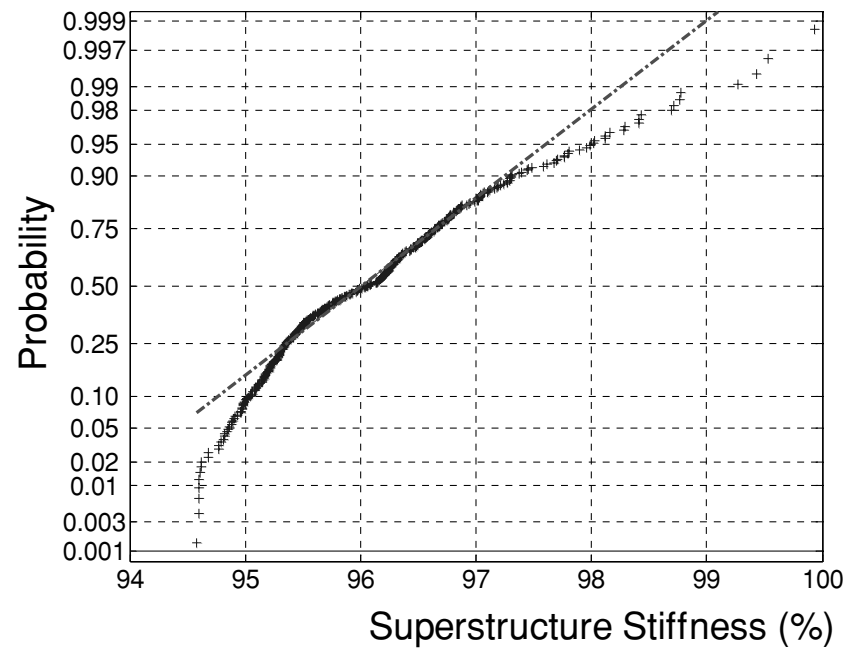

Fig. 12. Normal probability plot for the superstructure stiffness identification results.

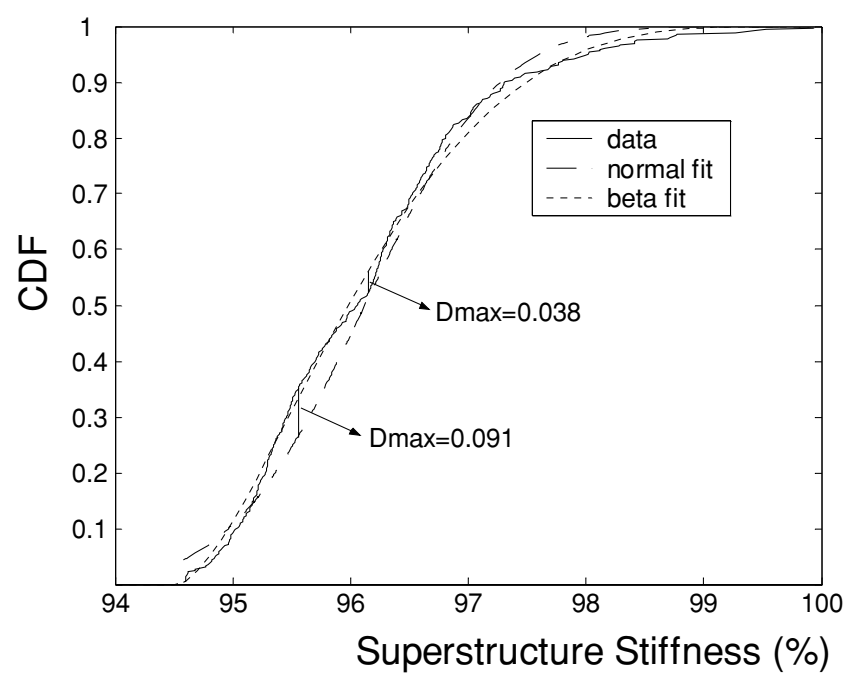

Fig. 13. K-S test for the Normal and Beta distributions.

Figure 15 shows the modes of each distribution for the summer and winter period over a 5-year period. Average of the best linear fits to summer and winter periods is given in Equation (7). A clear trend is observed indicating a decrease in the superstructure stiffness each year.

$$
\text { Stiffness }=96.2-0.4 * \text { years }
$$

Based on Equation (7), 0.4\% decrease in the superstructure stiffness per year can be estimated which constitutes $2 \%$ change over a 5 -year period. As stated in Section 2, monitoring of the bridge was started 4 years after the bridge was opened to the traffic. It is likely that changes in structural stiffness due to the pre-stress 


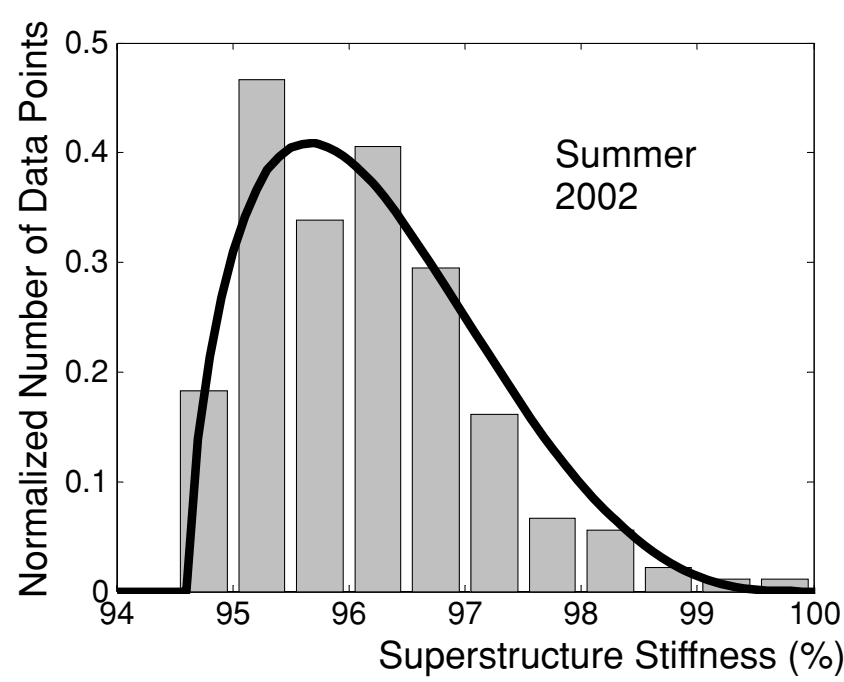

Fig. 14. Probability distribution of superstructure stiffness values.

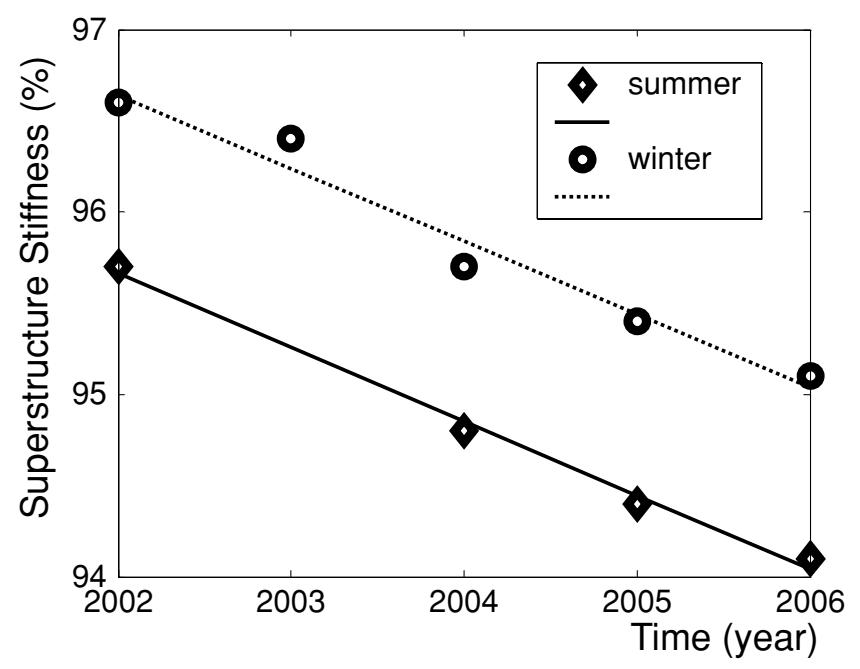

Fig. 15. Trend in superstructure stiffness values.

loss had stabilized in the first 4 years. Therefore the identified decrease in the stiffness value is not due to pre-stress loss. The degradation of the stiffness is considered due to material deterioration over the monitoring period.

\section{CONCLUSION}

This article presents vibration monitoring results of a new concrete bridge over a 5-year period. The real-time wireless data transmission capability of the sensor system on the bridge facilitates convenient data collection in the office. In total, 1,707 traffic-induced vibration data sets were collected during a 5 -year period, constituting a valuable database. Based on the vibration data, the bridge structural parameters including mass and stiffness, as well as modal parameters, were identified. From the modal and structural parameter identification and the statistical analysis, the following observations can be made:

1. Over the 5-year monitoring period, a 5\% decrease in the first modal frequency and a $2 \%$ decrease in the superstructure stiffness were observed.

2. Over the same period, a fluctuation between $-10 \%$ and $+10 \%$ was observed in the first modal frequency, while a fluctuation between $-3 \%$ and $+3 \%$ was observed in the superstructure stiffness.

3. Probability density functions for the structural stiffness values were proposed. The distributions themselves together with the decrease in the representative values of these distributions can be used to assess the time-varying reliability of the bridge structure.

This study represents the first step in developing an analytical tool for the condition assessment of the bridge superstructure using stiffness values. The methods developed by this study provide a tool that can be used for, for example, the FHWA's Long-Term Bridge Performance Program.

\section{ACKNOWLEDGMENTS}

This study was sponsored by Caltrans under the research grant 59A0311. The authors are thankful for insightful comments by project manager Mr. Li-Hong Sheng.

\section{REFERENCES}

Adeli, H. \& Karim, A. (2000), Fuzzy wavelet RBFNN model for freeway incident detection, Journal of Transportation Engineering, ASCE, 126(6), 464-71.

Adeli, H. \& Karim, A. (2005), Wavelets in Intelligent Transportation Systems, Haboken, New Jersey.

Akgul, F. \& Frangapol, D. M. (2004), Lifetime performance analysis of existing prestressed concrete bridge superstructures, Journal of Structural Engineering, ASCE, 130(12), 1889-903.

Beck, J. L. \& Jennings, P. C. (1980), Structural identification using linear models and earthquake records, Earthquake Engineering and Structural Dynamics, 8(2), 145-60.

Brinker, R., Zhang, L. \& Andersen, P. (2001), Modal identification of output-only system using frequency domain decomposition, Smart Materials and Structures, 10(3), 441-55.

Calcada, R., Cunha, A. \& Delgado, R. (2005), Analysis of traffic-induced vibrations in a cable-stayed bridge. Part II: numerical modeling and stochastic simulation, Journal of Bridge Engineering, 10(4), 386-97. 
Choi, S., Park, S., Bolton, R., Stubbs, N. \& Sikorsky, C. (2004), Periodic monitoring of physical property changes in a concrete box-girder bridge, Journal of Sound and Vibration, 278, 365-81.

Doebling, S. W., Farrar, C. R., Prime, M. B. \& Shevitz, D. W. (1996), Damage identification and health monitoring of structural and mechanical systems from changes in their vibration characteristics: A literature review, Los Alamos National Laboratory Report, LA-13070-MS.

Enright, M. P. \& Frangapol, D. M. (1998), Service-life prediction of deteriorating concrete bridges, Journal of Structural Engineering, 124(3), 309-17.

Federal Highway Administration (FHWA) (1995), Recording and Coding Guide for the Structure Inventory and Appraisal of the Nation's Bridges, U.S. Department of Transportation, Washington DC.

Federal Highway Administration (FHWA) (1996), Seismic design of bridges design example no. 6: Three-span continuous CIP concrete box bridge, Report No: FHWA-SA-97011, Washington DC.

Feltrin, G. (2002), Temperature and damage effects on modal parameters of a reinforced concrete bridge, Fifth European Conference on Structural Dynamics, EURODYN 2002, Munich, September 2-5, 373-78.

Feng, M. Q. \& Bahng, E.Y. (1999), Damage assessment of jacketed RC columns using vibration tests, Journal of Structural Engineering, 125(3), 265-71.

Feng, M. Q., Fukuda, Y., Chen, Y., Soyoz, S. \& Lee, S. (2006), Long-term structural performance monitoring of bridges, Technical Report of the California Department of Transportation, 2006-UCI-02.

Feng, M. Q., Kim, D. K., Yi, J. H. \& Chen, Y. B. (2003), Baseline models for bridge performance monitoring, Journal of Engineering Mechanics, 131(5), 562-69.

Feng, M. Q. \& Kim, J. M. (1998), Identification of a dynamic system using ambient vibration measurements, Journal of Applied Mechanics, 65(2), 1010-23.

Ghanem, R. \& Shinozuka, M. (1995), Structural system identification I: theory, Journal of Engineering Mechanics, 121(2), 255-64.

Green, M. F. \& Cebon, D. (1997), Dynamic interaction between heavy vehicles and highway bridges, Computers \& Structures, 62(2), 253-64.

Guan, H., Karbhari, V. M. \& Sikorsky C. S. (2006), WebBased structural health monitoring of an FRP composite bridge, Computer Aided Civil-Infrastructure Engineering, 21, 39-56.

Huang, C. C. \& Loh, C. H. (2001), Nonlinear identification of dynamic systems using neural networks, Computer-Aided Civil and Infrastructure Engineering, 16, 28-41.

Jiang, X. \& Adeli, H. (2007), Pseudospectra, MUSIC, and dynamic wavelet neural network for damage detection of highrise buildings, International Journal for Numerical Methods in Engineering, 71(5), 606-29.

Jiang, X. \& Adeli, H. (2008a), Dynamic fuzzy wavelet neuroemulator for non-linear control of irregular building structures, International Journal for Numerical Methods in Engineering, 74(7), 1045-66.

Jiang, X. \& Adeli, H. (2008b), Neuro-genetic algorithm for non-linear active control of structures, International Journal for Numerical Methods in Engineering, 75(7), 770-86.
Karim, A. \& Adeli, H. (2002), Comparison of the fuzzywavelet RBFNN freeway incident detection model with the California algorithm, Journal of Transportation Engineering, ASCE, 128(1), 21-30.

Karim, A. \& Adeli, H. (2003), Radial basis function neural network for work zone capacity and queue estimation, Journal of Transportation Engineering, ASCE, 129(5), 494503.

Lee, J. J., Lee, J. W., Yi, J. H., Yun, C. B. \& Jung, H. Y. (2005), Neural networks-based damage detection for bridges considering errors in baseline finite element models, Journal of Sound and Vibration, 280, 555-78.

Levin, R. I. \& Lieven, N. A. J. (1992), Dynamic finite element model updating using neural networks, Journal of Sound and Vibration, 210(5), 593-607.

Masri, S. F., Smyth, A. W., Chassiakos, A. G., Caughey, T. K. \& Hunter, N. F. (2000), Application of neural networks for detection of changes in nonlinear systems, Journal of Engineering Mechanics, 126(7), 666-76.

National Bridge Inspection Standards (NBIS) (1996), Code of federal regulations, No. 23CFR650, U.S. Government Printing Office, Washington DC., 238-40.

Otte, D., Ponseele, P. V. D. \& Leuridan, J. (1990), Operational shapes estimation as a function of dynamic loads, in Proceedings of 8th International Modal Analysis Conference, Society for Experimental Mechanics, Orlando, FL, 413-21.

Pan, T. C. \& Li, J. (2002), Dynamic vehicle element method for transient response of coupled vehicle-structure systems, Journal of Structural Engineering, 128(2), 214-23.

Peeters, B. \& DeRoeck, G. (2001), One-year monitoring of the Z24-Bridge: environmental effects versus damage events, Earthquake Engineering and Structural Dynamics, 30(2), 149-71.

Phares, B. M., Washer, G. A., Rolander, D. D., Graybeal, B. A. \& Moore, M. (2004), Routine highway bridge inspection condition documentation accuracy and reliability, Journal of Bridge Engineering, 9(4), 403-13.

Safak, E. (1989), Adaptive modeling, identification and control of dynamic structural systems. I: theory, Journal of Engineering Mechanics, 115(11), 2386-405.

Safak, E. (1991), Identification of linear structures using discrete-time filters, Journal of Structural Engineering, 17(10), 3064-85.

Shinozuka, M. \& Ghanem, R. (1995), Structural system identification II: experimental verification, Journal of Engineering Mechanics, 121(2), 265-73.

Sohn, H., Dzwonczyk, M., Straser, E., Kiremidjian, A. S., Law, K. H. \& Meng, T. (1999), An experimental study of temperature effect on modal parameters of the Alamosa Canyon bridge, Earthquake Engineering and Structural Dynamics, 28(8), 879-97.

Stewart, M. G. \& Rosowsky, D. V. (1998), Time-dependent reliability of deteriorating reinforced concrete bridge decks, Structural Safety, 20, 91-109.

Yang, Y. B. \& Yau, J. D. (1997), Vehicle-bridge interaction element for dynamic analysis, Journal of Structural Engineering, 123(11), 1512-18.

Yun, C. B., Yi, J. H. \& Bahng E. Y. (2001), Joint damage assessment of framed structures using a neural networks technique, Engineering Structures, 23(5), 425-35. 